\title{
Changes in Stem Cell Transplant activity and procedures during SARS-CoV2 pandemic in Italy: an Italian Bone Marrow Transplant Group (GITMO) nationwide analysis (TransCOVID-19 Survey)
}

\author{
Domenico Russo $^{1}$ - Nicola Polverelli $\mathbb{1}^{1} \cdot$ Michele Malagola ${ }^{1} \cdot$ Mirko Farina $\mathbb{D}^{1}$ - Alessandro Leoni ${ }^{1}$ • \\ Simona Bernardi ${ }^{1,2} \cdot$ Sonia Mammoliti ${ }^{3}$ Nicoletta Sacchi ${ }^{4} \cdot$ Massimo Martino $\mathbb{1}^{5} \cdot$ Fabio Ciceri $\mathbb{1}^{6}$. \\ on behalf of GITMO Centers
}

Received: 16 January 2021 / Revised: 9 March 2021 / Accepted: 25 March 2021 / Published online: 19 April 2021

(c) The Author(s), under exclusive licence to Springer Nature Limited 2021

\begin{abstract}
The Transplant Centers belonging to Gruppo Italiano per il Trapianto di Midollo Osseo (GITMO) conducted a survey with the aim of evaluating the effect of SARS-CoV2 pandemic on the allogeneic transplant activity in Italy. The pandemic period from $1 / 3 / 2020$ to $31 / 7 / 2020$ was compared with the same period in 2019. Overall, in 2020 there was a $2.4 \%$ reduction in the number of allo-HCT cases compared to 2019. Interestingly, this deflection did not affect the acute leukemia cases $(+5.7 \%$ in 2020). The use of peripheral blood-derived stem cells $(+10.7 \%)$ and cryopreservation $(97.4 \%$ of the centers) was highly adopted in 2020. Despite the sanitary emergency, almost all of the surveyed centers declared no impact of SARS-CoV2 pandemic on the transplant timing and outcomes, and the sanitary policy was positively evaluated by the majority of centers. The emergency measures ensured that only a minority of the allo-HCT patients had been infected by SARS-CoV2; however, a mortality of $42.1 \%$ among the allo-HCT patients hospitalized for COVID-19 was recorded. This survey gives us the information that the GITMO Group reacted positively to the pandemic. Thanks to the emergency strategies, the Italian alloHCT activity continued safely, showing only a minor deflection and offering the same probability of cure to the transplanted patients.
\end{abstract}

The SARS-CoV2 pandemic dramatically affected the health activities. In Italy, as well as in the majority of the Countries, efforts have been made to guarantee treatments for acute and

Members of the GITMO Centers are listed above reference list.

These authors contributed equally: Domenico Russo, Nicola Polverelli

Supplementary information The online version contains supplementary material available at https://doi.org/10.1038/s41409021-01287-w.

$\triangle$ Domenico Russo

domenico.russo@unibs.it

$\triangle$ Nicola Polverelli

nicola.polverelli@unibs.it

1 Unit of Blood Diseases and Bone Marrow Transplantation, Cell Therapies and Hematology Research Program, Department of Clinical and Experimental Sciences, University of Brescia, ASST Spedali Civili di Brescia, Brescia, Italy

2 Centro di Ricerca Emato-Oncologica AIL (CREA), ASST Spedali oncological patients. This is the case of onco-hematological patients submitted or candidate to allogeneic hematopoietic stem cell transplantation (allo-HCT) [1]. Starting from 24th February, 2020, date on which the first COVID-19-positive patient has been identified in Italy, decrees and recommendations have been released by the National and International Health Authorities in order to provide adequate medical assistance to hematological and transplant patients [2] (http://www.salute.gov.it/portale/home.html, https://www. ebmt.org/covid-19-and-bmt, https://www.siematologia.it/ files/COVID19-Raccomandazioni-SIE-GITMO.pdf,

Civili di Brescia, Brescia, Italy

3 GITMO Trials Office, Genova, Italy

4 Italian Bone Marrow Donor Registry, Galliera Hospital, Genova, Italy

5 Stem Cell Transplant Program, Clinical Section, Department of Hemato-Oncology and radiotherapy, Grande Ospedale Metropolitano "Bianchi-Melacrino-Morelli”, Reggio Calabria, Italy

6 San Raffaele Scientific Institute, Milan, Italy 
Table 1 Comparison of transplant characteristics between March-July 2019 and March-July 2020.

\begin{tabular}{|c|c|c|c|c|}
\hline & \multirow[t]{2}{*}{$2019 \mathrm{~N}^{\circ}$} & \multirow[t]{2}{*}{$2020 \mathrm{~N}^{\circ}$} & \multicolumn{2}{|c|}{ Variation } \\
\hline & & & $\mathrm{N}^{\circ}$ & $\%$ \\
\hline Number of allo-HCT, $n(\%)$ & 462 & 451 & -11 & -2.4 \\
\hline \multicolumn{5}{|l|}{ Disease: } \\
\hline $\begin{array}{l}\text { Acute Leukemia (AML } \\
\& \text { ALL) }\end{array}$ & 281 & 297 & +16 & +5.7 \\
\hline $\begin{array}{l}\text { Myelodysplastic } \\
\text { Syndrome }\end{array}$ & 41 & 40 & -1 & -2.4 \\
\hline $\begin{array}{l}\text { Myeloproliferative } \\
\text { Neoplasms }\end{array}$ & 35 & 33 & -2 & -5.7 \\
\hline Hodgkin Lymphoma & 14 & 13 & -1 & -7.1 \\
\hline Non-Hodgkin Lymphoma & 41 & 26 & -15 & -36.6 \\
\hline Multiple Myeloma & 12 & 9 & -3 & -25.0 \\
\hline $\begin{array}{l}\text { Non-neoplastic } \\
\text { hematological diseases }\end{array}$ & 38 & 34 & -4 & -10.5 \\
\hline Oncological neoplasia & 0 & 1 & +1 & NA \\
\hline \multicolumn{5}{|l|}{ Type of Donor: } \\
\hline Sibling & 108 & 112 & +4 & +3.7 \\
\hline Unrelated Donor & 218 & 216 & -2 & -0.9 \\
\hline Haploidentical & 127 & 116 & -11 & -8.6 \\
\hline Cord Blood & 9 & 9 & 0 & 0 \\
\hline \multicolumn{5}{|l|}{ Source HSC: } \\
\hline Peripheral Blood & 339 & 376 & +37 & +10.0 \\
\hline Bone Marrow & 114 & 66 & -48 & -42.1 \\
\hline Cord Blood & 9 & 9 & 0 & 0 \\
\hline $\begin{array}{l}\text { Number of unrelated donor } \\
\text { searches }\end{array}$ & 405 & 412 & +7 & +1.7 \\
\hline $\begin{array}{l}\text { Shift from Foreign to } \\
\text { Italian Donor }\end{array}$ & 1 & 16 & +15 & +1500 \\
\hline Donor IC withdrawal & 4 & 10 & +6 & +150 \\
\hline Frontline Haplo transplant & 33 & 37 & +4 & +12.1 \\
\hline $\begin{array}{l}\text { Unrelated Donor not } \\
\text { available }\end{array}$ & 4 & 12 & +8 & +200 \\
\hline
\end{tabular}

IC informed consent, $N A$ not available.

http://www.ptpl.altervista.org/burl/2020/burl_33_2020_ddg_ 06082020_9583.pdf, https://www.ebmt.org/sites/default/ files/2020-03/EBMT\%20COVID-19\%20guidelines\%20v.3. 2\%20\%282020-03-16\%29.pdf). The most relevant ones allowed the transfer of a donor search from the international to the national bank registry (IBMDR) and the possibility to cryopreserve hematopoietic stem cells (HSCs), when there was concern that the donor was at high risk of communityacquired infection between work-up and collection (Supplementary Table 1).

On behalf of Gruppo Italiano per il Trapianto di Midollo Osseo (GITMO), we designed a specific Survey (TransCOVID-19 Survey), consisting of 40 multiple choices questions in order to investigate the effects of SARS-CoV2 pandemic on the Italian Transplant Centers activity. The first pandemic wave (from March 2020 the $1^{\text {st }}$ to July 2020 the $31 \mathrm{st}$ ) was compared to the same period of the year 2019 . Five different items were investigated: (I) the transplant activity in terms of numbers and characteristics of transplant procedures; (II) the opinion of Centers regarding transplant outcome in COVID-19 era; (III) the number of subjects infected by SARS-CoV2 virus among allo-HCT patients and staff personnel; (IV) the future transplant perspectives, and $(\mathrm{V})$ the grade of satisfaction about the National and International recommendations.

The survey was completed by a total of 39/47 (83.0\%) allogeneic transplant Centers, with $100 \%$ valid answers. In addition, eight GITMO-affiliated autologous transplant Centers sent information on the total number of autologous transplant procedures in order to evaluate the impact of the pandemic in the autologous setting. Overall, they declared 51 auto-transplant in 2020 compared to 60 in $2019(-15 \%$ decrease). No more analyses were carried out in autotransplant category (Supplementary Tables 2 and 3a-e).

Taking into account the Italian allo-HCT activity, there was only a mild decline in the numbers of allo-HCT compared to the same period of the previous year $(-2.4 \%)$ (Table 1). Interestingly, the reduction mostly affected less aggressive diseases, such as lymphoma and myeloma, as well as autologous procedures, while an increase in the number of allo-HCT for acute leukemias $(+5.7 \%)$ was even observed. The reason for this observation may be wellexplained by the different allo-HCT urgency of such diseases [3, 4]. The effect of SARS-CoV2 pandemic did not apparently impact on the algorithm of donor' choice. Indeed, in the large majority of cases, the Centers did not change their donor selection policies: sibling, unrelated, haploidentical and cord blood stem cell source distribution slightly changed during the pandemic. Nevertheless, more frontline haploidentical donors were reported and a higher proportion of unrelated donors (UD) refused to donate for potential donation-related infectious risk. In order to maintain the donor selection and transplant standards, while ensuring the safety of patients undergoing conditioning therapy in case of unexpected donor unavailability, more UD were recruited by IBMDR and a significant increase in the use of peripheral blood-derived stem cells (PBSC) $(+10.9 \%)$ and PBSC cryopreservation (employed in 38 [97.4\%] in 2020 vs 19 Centers [48.7\%] in 2019) was observed, as recommended by the Health Authorities [5, 6].

Besides the raw numbers of transplant procedures, 23 centers $(59.0 \%)$ retained that SARS-CoV2 pandemic had no impact on transplant timing, while only 2 Centers (5.1\%) declared mild to significant reduction of transplant timing and $14(35.9 \%)$ an increase in the interval from the first patient evaluation to transplant. Altogether, these observations may reflect the efforts of allo-HCT transplant Centers 
to guarantee the transplant continuity. In doing so, whilst in 2019 almost all Center $(35 / 39,89.7 \%)$ did not use telemedicine, 31 centers $(79.5 \%)$ declared telemedicine employment during the pandemic: notably, in 14 out of 31 centers $(45.2 \%)$ telemedicine was extended to $\geq 25 \%$ of ambulatory visits. Moreover, in the opinion of the large majority of the interviewed Centers, there was no effect of the pandemic on the transplant-related outcomes.

Eighteen allo-HCT Centers $(46.2 \%)$ managed hematological patients with COVID-19. The rate of hematological SARS-CoV2 positive patients was generally low (1-9 cases for $88,9 \%$ of involved Centers). However, among the hospitalized allo-HCT SARS-CoV2-positive patients the mortality rate was particularly high (8 cases out of $19,42.1 \%$ ). In 9 Transplant Centers $(23.1 \%)$ personnel redistribution was requested to provide assistance to non-hematologic SARS-CoV2-positive patients. Regarding SARS-CoV2infected personnel, 22 Centers $(56.4 \%)$ referred staff members to be infected by SARS-CoV2 virus, for a total of 79 individuals. Seven out of 79 subjects $(8.9 \%)$ required hospitalization in three centers $(7.7 \%)$ with no fatalities. The isolation measures well limited the spread of the infection among allo-HCT patients. Nevertheless, the virus confirmed its severe impact on immune-depressed patients, with a mortality higher than $40 \%$ among the hospitalized transplant subjects, as recently reported by an Italian multicenter study [7]. Also, SARS-CoV2 pandemic had a significant impact on the staff personnel too, highlighting once again the psychological and physical implications of the pandemic among the hospital professionals.

The latter part of the survey relied on possible transplant perspectives after the end of SARS-CoV2 crisis. For 28 Centers $(71.8 \%)$, an extended PBSC utilization could be maintained or even increased in the early future; on the contrary, PBSC cryopreservation was expected to return to pre-SARS-CoV2 era by $16(41.0 \%)$ Centers, while 13 Centers $(33.3 \%)$ and 10 Centers $(25.6 \%)$ foresaw only a mild decrease or stabilization. For 28 (71.8\%) Centers the preferential use of Nationals donors compared to foreign donors will be maintained $(15,38.5 \%)$ or even increased $(13,33.3 \%)$. A total 16 Centers $(41.0 \%)$ predicted a stabilization or increase in the use of telemedicine, while 12 $(30.8 \%)$ and $11(28.2 \%)$ Centers hypothesized a slight or significant reduction, respectively.

Finally, the Centers reported a positive judgement of indications and recommendations provided by Italian and European Health Authorities; overall, as confirmed by the first Survey's section, the recommendations effectively guided the Italian transplant activity by all the surveyed Centers.

What is going to remain after this experience in the future? The opinion poll considered the emergency measures as the cryopreservation of HSCs, the use of telemedicine useful but temporary in the majority of cases.
Long-term analyses are required to confirm the safety and efficacy of such approach in normal clinical practice.

In conclusion, this survey suggests that collaborative network between Health Authorities, Scientific Societies and Transplant Centers ensured to continue a safe transplant activity among GITMO-affiliated Centers.

GITMO Centers Francesco Zallio $^{7}$, Attilio Olivieri ${ }^{8}$, Sadia Falcioni ${ }^{9}$, Gabriella Storti ${ }^{10}$, Mariagrazia Michieli ${ }^{11}$, Paola Carluccio ${ }^{12}$, Anna Grassi $^{13}$, Elena Oldani ${ }^{13}$, Francesca Bonifazi ${ }^{14}$, Arcangelo Prete ${ }^{15}$, Irene Maria Cavattoni ${ }^{16}$, Marianna Maffeis ${ }^{17}$, Domenico Pastore ${ }^{18}$, Adriana Vacca ${ }^{19}$, Daniela Caravelli ${ }^{20}$, Milena Mirabile ${ }^{21}$, Nicola Mordini $^{22}$, Chiara Nozzoli ${ }^{23}$, Maura Faraci ${ }^{24}$, Vincenzo Federico ${ }^{25}$, Sonia Ronconi ${ }^{26}$, Cristina Skert ${ }^{27}$, Francesco Onida ${ }^{28}$, Magda Marcatti $^{29}$, Simona Piemontese ${ }^{29}$, Franco Narni ${ }^{30}$, Adriana Balduzzi ${ }^{31}$, Giuseppina De Simone ${ }^{32}$, Alessandra Picardi ${ }^{33}$, Marco De Gobbi ${ }^{34}$, Elisabetta Calore ${ }^{35}$, Stefania Tringali ${ }^{36}$, Marco Zecca ${ }^{37}$, Simona Secondino ${ }^{37}$, Barbara Guiducci ${ }^{38}$, Matteo Pelosini ${ }^{39}$, Eliana Zuffa ${ }^{40}$, Luca Facchini $^{41}$, Manuela Imola ${ }^{42}$, Anna Paola Iori ${ }^{43}$, Anna Proia ${ }^{44}$, Simona Sica ${ }^{45}$, Daniele Armiento ${ }^{46}$, Angelo Michele Carella ${ }^{47}$, Chiara Maria Dellacasa ${ }^{48}$, Franca Fagioli ${ }^{49}$, Marco Rabusin ${ }^{50}$, Andrea Ferrario $^{51}$, Francesca Elice ${ }^{52}$

${ }^{7}$ Unit of Hematology, AO SS Antonio e Biagio e Cesare Arrigo, Alessandria, Italy; ${ }^{8}$ Unit of Hematology, Azienda OspedalieroUniversitaria Ospedali Riuniti di Ancona, Ancona, Italy; ${ }^{9}$ Unit of Hematology, C. e G. Mazzoni Hospital, Ascoli Piceno, Italy; ${ }^{10}$ Unit of Hematology, Azienda Ospedaliera S. G. Moscati, Avellino, Italy; ${ }^{11}$ Bone Marrow Unit, C.R.O., Aviano, Italy; ${ }^{12}$ Unit of Hematology, Azienda ospedaliero-universitaria Policlinico, Bari, Italy; ${ }^{13}$ Unit of Hematology, ASST Papa Giovanni XXIII, Bergamo, Italy; ${ }^{14}$ Unit of Hematology, IRCCS Azienda Ospedaliero Universitaria di Bologna, Bologna, Italy; ${ }^{15}$ Department of Oncology and Pediatric Hematology, S.Orsola-Malpighi Hospital, Bologna, Italy; ${ }^{16}$ Unit of Hematology and BMT, Central Hospital, Bolzano, Italy; ${ }^{17}$ Pediatric Onco-Hematology and Bone Marrow Transplant (BMT) Unit, Children Hospital, Brescia, Italy; ${ }^{18}$ Unit of Hematology, Perrino Hospital, Brindisi, Italy; ${ }^{19}$ Unit of Hematology and BMT, Roberto Binaghi Hospital, Cagliari, Italy; ${ }^{20}$ Unit of Hematology, Candiolo IRCCS Hospital, Torino, Italy; ${ }^{21}$ Unit of Hematology, Area Vasta 3, Civitanova Marche, Italy; ${ }^{22}$ Unit of Hematology, AO Santa Croce e Carle, Cuneo, Italy; ${ }^{23}$ Unit of Hematology, Azienda OspedalieraUniversitaria Careggi, Firenze, Italy; ${ }^{24}$ Pediatric Hematology and Oncology, IRCCS Istituto G. Gaslini, Genova, Italy; ${ }^{25}$ Unit of Hematology, Vito Fazzi Hospital, Lecce, Italy; ${ }^{26}$ Unit of Hematology, IRCS IRCCS, Meldola, Italy; ${ }^{27}$ Unit of Hematology, Ospedale Dell'Angelo, Mestre-Venezia, Italy; ${ }^{28}$ Unit of Hematology and BMT, Policlinico di Milano, Milano, Italy; ${ }^{29}$ Unit of Hematology and BMT, San Raffaele Hospital, Milano, Italy; ${ }^{30}$ Unit of Hematology and BMT, Policlinico di Modena, Modena, Italy; ${ }^{31}$ Pediatric Hematology and BMT, ASST Monza, Monza, Italy; ${ }^{32}$ Pediatric Hematology and BMT, Santobono-Pausilipon Hospital, Napoli, Italy; ${ }^{33}$ Unit of Hematology and BMT, Cardarelli Hospital, Napoli, Italy; ${ }^{34}$ Unit of Hematology and BMT, San Luigi Gonzaga Hospital, Orbassano, Italy; ${ }^{35} \mathrm{Clinica}$ Onco-Ematologia Pediatrica, Ospedale di Padova, Padova, Italy; ${ }^{36}$ Unit of BMT, Azienda ospedaliera Villa Sofia-Cervell, Palermo, Italy; ${ }^{37}$ Fondazione IRCCS Policlinico San Matteo, Pavia, Italy; ${ }^{38}$ Unit of Hematology and BMT, Ospedali Riuniti Marche Nord, Pesaro, Italy; ${ }^{39}$ Clinical and Experimental Medicine, Section of Hematology, Pisa, Italy; ${ }^{40}$ Unit of Hematology, Romagna Transplant Center, Hospital of Ravenna, Ravenna, Italy; ${ }^{41}$ Unit of Hematology, IRCCS Reggio Emilia, Reggio Emilia, Italy; ${ }^{42}$ Unit of Hematology, Infermi Hospital, Rimini, Italy; ${ }^{43}$ Department of Cellular Biotechnologies and Hematology, Sapienza University, Roma, Italy; ${ }^{44}$ Unit of 
Hematology and BMT, Azienda Ospedaliera S. Camillo-Forlanini, Roma, Italy; ${ }^{45}$ Unit of Hematology and BMT, Fondazione Policlinico Universitario A. Gemelli, Roma, Italy; ${ }^{46}$ Unit of Hematology and BMT, University Campus Bio-Medico, Roma, Italy; ${ }^{47}$ Unit of Hematology and BMT, Fondazione IRCCS "Casa Sollievo della Sofferenza", San Giovanni Rotondo, Italy; ${ }^{48}$ Unit of BMT, Molinette Hospital, Torino, Italy; ${ }^{49}$ Pediatric Onco-Hematology, Stem Cell Transplantation, and Cellular Therapy Division, Turin Metropolitan Transplant Center, A.O.U. Citta'della Salute e della Scienza, Torino, Italy; ${ }^{50}$ Institute for Maternal and Child Health, IRCCS Burlo Garofolo, Trieste, Italy; ${ }^{51}$ Unit of Hematology, ASST Sette Laghi, Varese, Italy; ${ }^{52}$ Pediatric Onco-Hematology, ULSS 8 Berica, Vicenza, Italy

\section{Compliance with ethical standards}

Conflict of interest The authors declare no competing interest.

Publisher's note Springer Nature remains neutral with regard to jurisdictional claims in published maps and institutional affiliations.

\section{References}

1. Malagola M, Polverelli N, Gandolfi L, Zollner T, Bernardi S, Zanaglio $\mathrm{C}$, et al. Severe acute respiratory syndrome coronavirus-2 pandemia: facts and perspectives in a bone marrow transplant unit. Front Oncol. 2020;10:1626.
2. Ljungman P, Mikulska M, de la Camara R, Basak GW, Chabannon C, Corbacioglu S, et al. The challenge of COVID-19 and hematopoietic cell transplantation; EBMT recommendations for management of hematopoietic cell transplant recipients, their donors, and patients undergoing CAR T-cell therapy. Bone Marrow Transplant. 2020;55:2071-2076.

3. Morello E, Malagola M, Bernardi S, Pristipino C, Russo D. The role of allogeneic hematopoietic stem cell transplantation in the four P medicine era. Blood Res 2018;53:3-6.

4. Brissot E, Labopin M, Baron F, Bazarbachi A, Bug G, Ciceri F, et al. Management of patients with acute leukemia during the COVID-19 outbreak: practical guidelines from the acute leukemia working party of the European Society for Blood and Marrow Transplantation. Bone Marrow Transplant. 2021;56:532-535.

5. Ardura M, Hartley D, Dandoy C, Lehmann L, Jaglowski S, Auletta JJ. Addressing the impact of the coronavirus disease 2019 (COVID-19) pandemic on hematopoietic cell transplantation: learning networks as a means for sharing best practices. Biol Blood Marrow Transplant 2020;26:e147-e160.

6. Mengling T, Rall G, Bernas SN, Astreou N, Bochert S, Boelk T, et al. Stem cell donor registry activities during the COVID-19 pandemic: a field report by DKMS. Bone Marrow Transplant. 2020;1-9. https://doi.org/10.1038/s41409-020-01138-0.

7. Passamonti F, Cattaneo C, Arcaini L, Bruna R, Cavo M, Merli F, et al. Clinical characteristics and risk factors associated with COVID-19 severity in patients with haematological malignancies in Italy: a retrospective, multicentre, cohort study. Lancet Haematol. 2020;7:e737-e745. 